\title{
Một số nghiên cứu tiêu biểu dẫn chiếu cộng tính văn hóa
}

\author{
Nguyễn Thanh Thanh Huyền \\ April 17, 2021

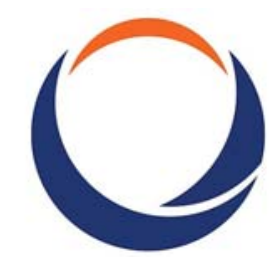 \\ PHENIKAA \\ UNIVERSITY \\ Trung tâm ISR \\ Trường Đại học Phenikaa \\ Yên Nghĩa, Hà Đông, Hà Nội, Việt Nam
}

Cộng tính văn hóa là khái niệm được sinh ra và định nghĩa trong nghiên cứu xuất bản vào tháng 12 năm 2018, trên tạp chí Palgrave Communications (ISSN 2662-9992, tạp chí khoa học xã hội nhân văn duy nhất được xuất bản trên nature.com, và được đổi tên thành Humanities \& Social Sciences Communications kể từ tháng 7 năm 2020). Có thể truy cập và đọc toàn văn bài nghiên cứu tại địa chỉ Internet: https://www.nature.com/articles/s41599-018-0189-2.

Bài nghiên cứu "Cultural additivity: behavioural insights from the interaction of Confucianism, Buddhism and Taoism in folktales" cũng là một trong những sản phẩm được xem như tiên phong trong sử dụng phương pháp thống kê Bayesian, dẫn tới việc ra đời của chương trình bayesvl trong môi trường ngôn ngữ tính toán thống kê $\mathrm{R}$ của Trung tâm ISR (La \& Vuong, 2019).

Ý niệm cộng tính văn hóa và cơ chế của nó là một ứng dụng lý thú của lý thuyết "mindsponge", do tác giả Vương Quân Hoàng đề xướng năm 2013, và chính thức công bố quốc tế các năm 2015 và 2016 (Vuong, 2016; Vuong \& Napier, 2015), vài ba năm trước khi Trung tâm ISR ra đời.

Điều đáng khích lệ với khái niệm "cộng tính văn hóa” (cultural additivity) là không bao lâu sau khi được xuất bản chính thức, cả khái niệm lẫn cơ chế giải thích sự hình 
thành nên nó, cũng như ảnh hưởng của nó, đã bắt đầu được công nhận và sử dụng. Có thể điểm nhanh một số dẫn chiếu sử dụng tiêu biểu:

Bảng 1. Một số tác phẩm sử dẫn chiếu tới khái niệm cộng tính văn hóa

\begin{tabular}{|l|l|l|}
\hline Tác giả (năm) & Lĩnh vực & Án phẩm \\
\hline Bay (2020) & Internet ethics & $\begin{array}{l}\text { AolR Selected Papers of Internet } \\
\text { Research }\end{array}$ \\
\hline Dos Santos (2020) & Healthcare & $\begin{array}{l}\text { International Journal of Environmental } \\
\text { Research and Public Health }\end{array}$ \\
\hline $\begin{array}{l}\text { Giang-Le, Hsiao \& Heo } \\
(2020) .\end{array}$ & Education & $\begin{array}{l}\text { International Journal for Cross- } \\
\text { Disciplinary Subjects in Education }\end{array}$ \\
\hline $\begin{array}{l}\text { Konno \& Schillaci } \\
(2021)\end{array}$ & $\begin{array}{l}\text { Innovation / } \\
\text { Knowledge } \\
\text { Management }\end{array}$ & Sournal of Intellectual Capital \\
\hline $\begin{array}{l}\text { Le \& Yu (2021) } \\
\text { (2020) }\end{array}$ & University research & $\begin{array}{l}\text { Higher Education in Market-Oriented } \\
\text { Socialist Vietnam }\end{array}$ \\
\hline Nguyen et al. (2019) & Mental health & Transcultural Psychiatry \\
\hline Siebenhütter (2019) & Cultural knowledge & $\begin{array}{l}\text { Conceptual Transfer as an Areal } \\
\text { Factor }\end{array}$ \\
\hline Small \& Blanc (2020) & Mental health & Frontiers in Psychiatry \\
\hline $\begin{array}{l}\text { Stoermer, Lauring \& } \\
\text { Selmer (2020) }\end{array}$ & Creativity & $\begin{array}{l}\text { International Journal of Intercultural } \\
\text { Relations }\end{array}$ \\
\hline Nguyen (2019) & Emotional intelligence & $\begin{array}{l}\text { Doctoral dissertation at Bournemouth } \\
\text { University. }\end{array}$ \\
\hline Le \& Yu (2019) & Social research & Sexuality \& Culture \\
\hline
\end{tabular}

\section{(Danh sách chi tiết thông tin thư viện xin xem ở Tài liệu tham khảo)}

Trong số các tác giả trên, có cả những học giả rất nổi tiếng như Noboru Konno, Keio University, https://scholar.google.com/citations?user=uRPoHTYAAAAJ. Ông là đồng tác giả với một học giả lừng lẫy khác của Nhật Bản suốt từ những năm 1990 là Ikujiro Nonaka (Hitotsubashi University, https://en.wikipedia.org/wiki/lkujiro Nonaka). Hai tác giả này đã làm say mê nhiều thế hệ học giả và học sinh với các nghiên cứu về quản trị tri thức và quá trình sáng tạo/đổi mới trong doanh nghiệp, bao gồm cả những hãng công nghiệp thành công lừng lẫy của Nhật Bản thời kỳ hoàng kim, thập niên 1990s. 
Một học giả nữa cũng rất quen thuộc với giới nghiên cứu là Jan Selmer, Aarhus University, https://scholar.google.com/citations?user=tzt1zPkAAAAJ. Giáo su Selmer cũng được biết đến rộng rãi với vai trò Tổng biên tập tạp chí Journal of Global Mobility (ISSN 2049-8799) của Nhà xuất bản Emerald.

Tính đến thời điểm này, bản thảo sạch đầu tiên của nghiên cứu cộng tính văn hóa mới tròn 3 tuổi, và bài nghiên cứu xuất bản chính thức trên Palgrave Communications mới 2 tuổi 4 tháng. Tuy nhiên, mức độ chú ý và ảnh hưởng của tác phẩm thì cũng không còn được coi là rất mới mẻ nữa. Đó là nhờ ngày càng nhiều tác giả và tác phẩm có sức nặng, trên các ấn phẩm giá trị dẫn chiếu và sử dụng ý niệm này.

Những cán bộ nghiên cứu của Trung tâm ISR, Trường Đại học Phenikaa, ngoài mong muốn, thì cũng ngày càng có hy vọng và cơ sở để tin rằng, trong tương lai, mức độ ảnh hưởng của khái niệm và phương pháp tiếp cận cũng như ứng dụng của "cộng tính văn hóa" sẽ còn tiếp tục gia tăng trên hệ thống tư liệu học thuật quốc tế.

Khi điều này trở thành hiện thực, đối với Trung tâm ISR, đó không chỉ đơn giản là niềm vui và tự hào với kết quả lao động khoa học, mà còn đánh dấu bước trưởng thành của một lý thuyết sinh ra từ Việt Nam, do những con người của nước Nam đã tự tay triển khai, hoàn thành và thổi hồn tạo nên sức sống cho sản phẩm tri thức.

Nó cũng là sự nhắc nhở về truyền thống khoa học dù non trẻ, nhưng rất đáng khích lệ của Trung tâm ISR; góp phần động viên các thế hệ nghiên cứu trẻ trong tương lai vững bước tiến lên trong kỷ nguyên hội nhập khoa học toàn cầu.

\section{Tài liệu tham khảo:}

Bay, M. (2020). Google and Facebook vs. Rawls and Lao-Tzu: How Silicon Valley's Utilitarianism and Confucianism Are Bad for Internet Ethics. AolR Selected Papers of Internet Research.

Dos Santos, L. M. (2020). I am a nursing student but hate nursing: The East Asian perspectives between social expectation and social context. International Journal of Environmental Research and Public Health, 17(7), 2608.

Giang-Le, N. H., Hsiao, C. T., \& Heo, Y. (2020). Trans-Cultural Journeys of East-Asian Educators: The Impact of the Three Teachings. International Journal for CrossDisciplinary Subjects in Education, 11(1):4201-4210.

Kim, P. D. T. (2020). Barriers to Entrepreneurship in Ho Chi Minh City. Unpub. Master's Thesis, Tampere University.

Konno, N., \& Schillaci, C. E. (2021). Intellectual capital in Society 5.0 by the lens of the knowledge creation theory. Journal of Intellectual Capital, AOP. DOI:

10.1108/JIC-02-2020-0060. 
La, V. P., \& Vuong, Q. H. (2019). bayesvl: Visually learning the graphical structure of Bayesian networks and performing MCMC with 'Stan'. The Comprehensive $R$ Archive Network (CRAN).

Le, N., Hsiao, C. T., \& Heo, Y. (2019). Educator Identity Development on The Transcultural Journeys. 2019 Canadian International Conference on Education, Toronto. Toronto, Canada: University of Toronto. pp. 1-4.

Le, T. M., \& Yu, N. (2019). Ideological and philosophical underpinnings of attitudes toward sexual minorities in Vietnamese society. Sexuality \& Culture, 23(2), 444457.

Le, T. M., \& Yu, N. (2021). Vietnamese social work practitioners' conceptions of practice with sexual minorities. Qualitative Social Work, AOP. DOI: $10.1177 / 1473325021990874$.

Marklein, M. B., \& Van Tinh, M. (2020). The Construction, Deconstruction, and Reconstruction of Academic Freedom in Vietnamese Universities. In: Higher Education in Market-Oriented Socialist Vietnam (pp. 207-226). Palgrave Macmillan, Cham.

Nguyen, M. H., Lanca, J. C., Hahn, E., von Poser, A., Heyken, E., Wingenfeld, K., ... \& Ta, T. M. T. (2019). Migration-related emotional distress among Vietnamese psychiatric patients in Germany: An interdisciplinary, mixed methods study. Transcultural Psychiatry, 1363461520920329.

Nguyen, Q. (2019). Exploring emotional intelligence: a study of Vietnamese hotel workers. Doctoral dissertation, Bournemouth University.

Siebenhütter, S. (2019). Conceptual Transfer as an Areal Factor. In: Conceptual Transfer as an Areal Factor. De Gruyter Mouton.

Small, S., \& Blanc, J. (2020). Mental health during COVID-19: Tam Giao and Vietnam's response. Frontiers in Psychiatry, 11, 589618.

Stoermer, S., Lauring, J., \& Selmer, J. (2020). The effects of positive affectivity on expatriate creativity and perceived performance: What is the role of perceived cultural novelty?. International Journal of Intercultural Relations, 79, 155-164.

Tran, H. T. N. (2019). English language learner autonomy in the Vietnamese higher education context: enabling factors and barriers arising from assessment practice. Unpub. Doctoral Dissertation, School of Education, the University of Adelaide, Australia.

Vuong, Q. H. (2016) Global mindset as the integration of emerging socio-cultural values through mindsponge processes: A transition economy perspective. In: J. Kuada (ed.) Global Mindsets: Exploration and Perspectives (pp. 109-126). London: Routledge. 
Vuong, Q. H., et al. (2018). Cultural additivity: behavioural insights from the interaction of Confucianism, Buddhism and Taoism in folktales. Palgrave Communications, 4(1), 143.

Vuong, Q. H., \& Napier, N. K. (2015). Acculturation and global mindsponge: an emerging market perspective. International Journal of Intercultural Relations, 49, 354-367. 